\title{
Learning Structured Video Descriptions: Automated Video Knowledge Extraction for Video Understanding Tasks
}

\author{
Daniel Vasile and Thomas Lukasiewicz \\ Department of Computer Science, University of Oxford, UK \\ danielionut.vasile@gmail.com, thomas.lukasiewicz@cs.ox.ac.uk
}

\begin{abstract}
Vision to language problems, such as video annotation, or visual question answering, stand out from the perceptual video understanding tasks (e.g., classification) through their cognitive nature and their tight connection to the field of natural language processing. While most of the current solutions to vision-to-language problems are inspired from machine translation methods, aiming to directly map visual features to text, several recent results on image and video understanding have proven the importance of specifically and formally representing the semantic content of a visual scene, before reasoning over it and mapping it to natural language. This paper proposes a deep learning solution to the problem of generating structured descriptions for videos, and evaluates it on a dataset of formally annotated videos, which has been automatically generated as part of this work. The recorded results confirm the potential of the solution, indicating that it manages to describe the semantic content in a video scene with a similar accuracy to the one of state-of-the-art natural language captioning models.
\end{abstract}

Keywords: Structured video captioning - Video understanding.

\section{Introduction}

Video understanding represents one of the central problems in computer vision, and can be described as the task of automatically detecting and naming the objects in a scene, listing their attributes, and describing the relations between them. Several sub-problems are gathered under this generic task, such as video classification, video captioning, or visual question answering, the last two being part of the sub-category of vision to language problems. While video classification focuses on labelling videos based on their semantic content, video captioning aims to capture a video's semantics by generating a summarizing natural language sentence, and visual question answering involves reasoning over the semantic content of a video to answer a question formulated in natural language.

Recently, significant progress has been made towards understanding videos, with the current state-of-the-art models being able to classify and caption videos with natural language descriptions at close to human-level precision on certain datasets [22]. However, these models are still unable to logically reason about 
the semantics of a scene. A benchmark study on the current solutions for visual question answering identified that these performed poorly in situations requiring short-term memory (e.g., attribute comparisons), and that they struggled with long reasoning chains (such as count or existence questions) [8].

In a response to this, recent research in computer vision has been advocating for modelling the interactions and relationships between the objects in a scene using a formalized representation of these components [9]. This is based on the observation that reasoning using natural language is still a major open problem, while the task of reasoning in a formal setting (e.g., using ontology languages) has been extensively studied and is more approachable.

This paper tackles the general problem of video understanding by proposing three different models for learning structured descriptions that capture the semantic content of a video. These descriptions, formulated as RDF graphs, can be further expanded by leveraging commonsense and topic-specific knowledge available in Linked Data ontologies, and used to perform logical reasoning over the semantics of a video. We evaluate the proposed models on a semantically parsed video captioning dataset, and discuss the improvements that learning structured descriptions could bring to solving various video understanding tasks.

The motivation for our work on automatically describing videos in a structured manner stems from the very promising and impactful possible applications of such a solution. On the one hand, there is the potential of improving the reasoning abilities of the present state-of-the-art visual models, by abstracting away from the natural language currently used to describe video semantics. On the other hand, the wide availability of background information collected in the last decade in comprehensive knowledge bases such as DBpedia [2] and linguistic linked data graphs such as Framester [6], motivates devising general, domainindependent, and scalable video understanding solutions that harvest it. Lastly, the accelerated increase in the amount of video data across the internet, along with the potential of devising methods to structure and organise the information contained in it for later use in retrieval or question answering tasks, represents one more driving factor for this work.

Recent research indicates that explicitly extracting the semantics in a visual scene represents a promising direction to follow when solving vision to language problems. In [21], image captioning is addressed by first describing the image through a set of high-level concepts identified in it, and then using this representation to generate a summarizing sentence, to ultimately outperform the models that attempted to directly map image features to text. Moreover, being able to extract formal representations of the information inside videos would open the way towards mining commonsense and ontological knowledge from video data.

A natural step to take, once one is able to identify the semantic components in a visual scene, is to link them to entries in external knowledge-bases, in order to enrich the semantics extracted. To prove the usefulness of such a step, [19] presents a video captioning solution that detects the most relevant five objects in a scene, queries a knowledge base to obtain the semantic features describing these objects, and then maps this information to a summarizing natural language 
sentence. Their solution outperforms the more widely adopted video captioning models that only capture the knowledge present in their training datasets, highlighting the value of leveraging external knowledge. Furthermore, links to background information could also significantly impact the video semantics extraction phase, by giving the option of ranking and refining the automatically generated annotations through reasoning.

Finally, it is worth pointing out that devising a solution to comprehensively and formally annotate video content would significantly impact video understanding. From captioning, visual query answering, and action detection to video retrieval and learning from video input through knowledge extraction, all major fields of video understanding can benefit from such a solution.

The main contributions of this paper are summarized as follows:

- We provide a dataset ${ }^{1}$ of formally annotated videos. Since at the time of writing, no dataset of videos annotated in a formal language was publicly available, we first automatically devise such a dataset by semantically parsing to RDF graphs the natural language annotations in MSVD [3], a widely used video captioning dataset. The new dataset contains 1,970 video clips annotated by multiple RDF graphs composed of 4.3 triples on average (linked to entities in Framester [6]) and post-processed such that each represents a collection of facts formulated in the same underlying ontology.

- We develop a model based on deep neural networks to formally annotate videos. The main aim of this paper is the development of a machine learning model to produce formal descriptions for videos, structured as RDF graphs. We formulate the problem as multi-label classification, and address it using recent advances in deep neural video processing. We propose three learning models that map videos to structured annotations formulated as RDF graphs. The final test results, obtained by validating the proposed models on the newly devised dataset, indicate that they are able to describe the semantic content in a video scene with a similar accuracy to the one of state-of-the-art natural language captioning models.

To our knowledge, we are the first to provide a dataset of formally annotated videos and to develop a neural model to formally annotate videos.

The rest of this paper is organized as follows. Section 2 gives some preliminaries required to understand and contextualise our approach. Section 3 discusses the method used to devise datasets of formally annotated videos, and Section 4 presents the learning models employed to generate structured annotations. Section 5 describes the methodology used for validating and evaluating the proposed solution, and presents the annotation performance results recorded. Lastly, Section 6 concludes the paper, ending by setting possible directions for future work.

\footnotetext{
1 The dataset can be accessed on the authors' web pages.
} 


\section{Preliminaries}

We now briefly recall the basics of vision-to-language problems, deep-learning vision-to-language approaches, structured visual annotations, evaluating visionto-language performance, and evaluating structured annotation performance.

\subsection{Vision-to-Language Problems}

Vision-to-language problems stand at the intersection of video understanding and natural language processing, and have been the main focus of the recent work on automatically processing video content due to their impactful applications.

One of the central vision-to-language problems is video captioning, which aims to produce natural language sentences for describing video scenes. This goes beyond detecting the objects in the scene, or classifying it according to a set of labels, since it requires that the relationships between the participating entities are captured by the output description. Recently, there has been significant progress made on image captioning, most of the proposed solutions being based on deep-learning algorithms. When compared to image/frame captioning, describing videos poses the supplementary challenge of modelling spatio-temporal dynamics and relations. The task of visual query answering adds a reasoning component to video captioning, by requiring that the output sentence generated is the answer to an input query formulated in natural language.

\subsection{Deep-Learning Vision-to-Language Approaches}

The current state-of-the-art solutions to vision-to-language problems are based on deep neural architectures, taking advantage of recent breakthroughs in image analysis and machine translation. Most approaches in this category fit a common general setup described in Figure 1, aiming to project the semantics of both the textual query input $\mathbf{Q}$ (in the case of query answering tasks), and the video input $\mathbf{V}$ to a vector encoding $\mathbf{E}$ residing in a common space, to then decode these into a natural language sequence $\mathbf{S}$.

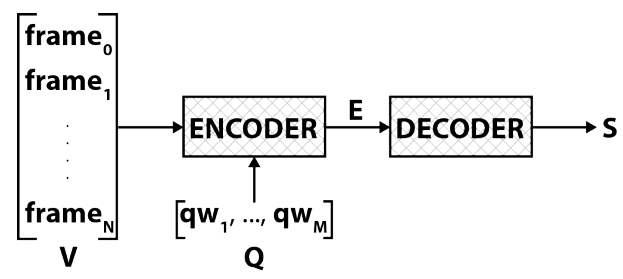

Fig. 1. General setup of a deep-learning vision-to-language model

The core of this type of systems, originally introduced for captioning images in [18], is an encoder-decoder setup inspired from machine translation. The input 
image is encoded using a CNN that is pre-trained on an image classification task; the fixed-length vector of the activations in the last hidden layer of the network is chosen as the image encoding. The decoding of this representation is achieved by passing it to an RNN that is trained to generate the captioning sequence of words one by one, at each step seeing the encoded image, as well as remembering the preceding generated words.

The main challenges of adapting such solutions to video annotation are handling variable-sized inputs, and addressing the temporal dynamics specific to sequences of frames. Figure 2 depicts the general structure of a deep-learning video encoder using LSTMs for capturing the frame-to-frame temporal variation. The role of the LSTM network is to provide a soft location attention [14] by focusing each encoding $\mathbf{e}_{i}$ onto the relevant parts of frame $\mathbf{e}_{i}$. The encoding of an optional query input is omitted in the diagram.

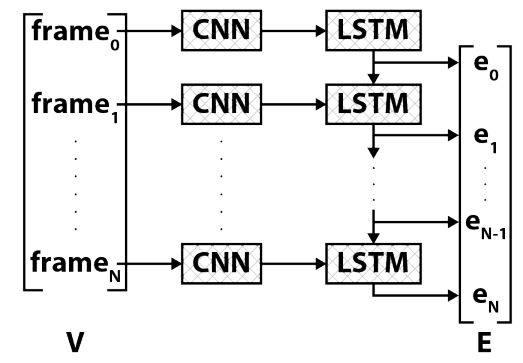

Fig. 2. General setup of a deep-learning video encoder

Due to the nature of video clips being of variable length, the generative RNN language model used for decoding cannot directly input E. Instead, the decoder for video captioning is augmented with an attention mechanism, which focuses on only a part of the encoding at each decoding step, producing fixedlength inputs for the language model. Figure 3 describes the approach to video captioning presented in [5].

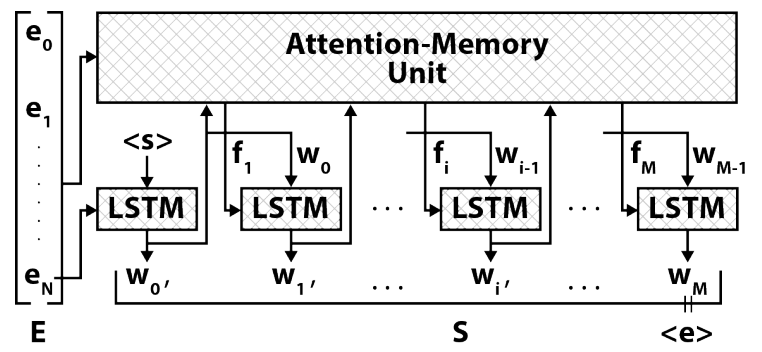

Fig. 3. Attention-Memory deep-learning video decoder presented in [5] 
The Attention-Memory Unit depicted in Figure 3 and introduced in [5] is an iterative memorized attention between the encoding $\mathbf{E}$ of an input video and the generated description $\mathbf{S}$, which learns where to attend in the video. It remembers encoded versions of the video and of the corresponding generated language (depicted by the $\mathbf{f}$ vectors in the diagram), allowing the decoding language model to selectively access the relevant information about the entire video and the previously generated words at each decoding step.

\subsection{Structured Visual Annotations}

As highlighted in the introduction, the current state-of-the-art vision-to-language models struggle to perform even simple logical reasoning, although they manage to achieve relatively high scores on visual question answering tasks. This might indicate that these models learn to exploit language biases in the benchmarking datasets [8], instead of learning to reason using visual and natural language inputs. A simpler, more abstract, and structured representation of the semantics in a visual scene may help to avoid the complexity of natural language when learning such models, and should improve their reasoning abilities.

Based on this observation, the Visual Genome dataset of formal dense image annotations is introduced in [9]. It aims to formally describe the components of each image, providing detailed annotations about the attributes and relations of each of the objects in the scene. The collection of objects in an image, their attributes, and relationships form a scene graph linked to WordNet synsets.

The annotations' density comes from the high average number of objects (35), attributes (26), and pairwise relationships between objects (21) per image, which significantly surpass the semantic complexity of the summary-level natural language captions in the standard image captioning datasets. The set of formal image annotations has been collected by employing an Amazon Mechanical Turk pipeline. To our knowledge, no similar dataset of structured video annotations was freely available at the time of writing.

\subsection{Evaluating Vision-to-Language Performance}

A series of metrics have been proposed for measuring the accuracy of visionto-language models. Most of these have been developed in the field of machine translation, and measure the similarity between two sequences by performing n-gram matching.

$B L E U[12]$ is an adapted n-gram precision metric that is designed to penalize predictions that achieve high precision by containing more repetitions of particular n-grams than in the set of reference sequences. $B L E U-n$ is measured as an weighted average of the BLEU scores for the i-grams with $i<=n$. METEOR [4]

is a unigram F-score that aligns the candidate sentence to a reference one by leveraging stemming and synonymy matching. Compared to BLEU, METEOR is evaluated for each reference in a corpus; the scores are aggregated by selecting the highest one. 
CIDEr [17] has been specifically proposed for measuring image captioning performance. It is designed to estimate the similarity between a predicted caption and a corpus of reference sentences by the sum of cosine similarities across ngrams, weighted by their term frequency-inverse document frequency (tf-idf) to reflect their informativeness.

While the metrics above have been shown to correlate well with human judgement on specific datasets (especially METEOR and CIDEr) [17], it is expected that only focusing on $n$-gram overlap (even when performing stemming and synonymy matching) is not enough to measure the semantic overlap between a predicted sequence and a corpus of reference ones.

SPICE [1] is a recent alternative metric focusing on measuring the semantic overlap and has been shown to correlate with human judgements better than BLEU, METEOR, and CIDEr. To measure the SPICE score, both the predicted and the reference captions are first semantically parsed into scene graphs using an adaptation of the rule-based Stanford Scene Graph Parser [13].

\subsection{Evaluating Structured Annotation Performance}

Measuring similarities between graphs of linked entities is usually achieved by comparing their structure and the semantic distance between entities given an underlying ontology. The authors of SPICE propose evaluating it as a specific F-score described below, in which entities are matched at WordNet synset level.

First, let $G(c)$ denote the scene graph obtained by parsing a predicted caption (or question answer) $c$, and let $G(S)$ be the union of the scene graphs $G\left(s_{i}\right)$ obtained by parsing the reference sequences $s_{i} \in S$. Next, define $T(G)$ to be the function that returns the union between the set of triples and the set of mentioned subjects and objects in the scene graph $G$. Finally, define precision P, recall $\mathrm{R}$, and the SPICE F-score as follows:

$$
\begin{gathered}
P(c, S)=\frac{|T(G(c)) \otimes T(G(S))|}{|T(G(c))|}, \\
R(c, S)=\frac{|T(G(c)) \otimes T(G(S))|}{|T(G(S))|}, \\
\operatorname{SPICE}(c, S)=\frac{2 P(c, S) R(c, S)}{P(c, S)+R(c, S)},
\end{gathered}
$$

where $\otimes$ performs the intersection of two sets of tuples by matching entities at WordNet synset level.

\section{Devising Datasets of Formally Annotated Videos}

In the absence of free datasets of formally annotated videos, and considering the significant cost of manually building a new dataset, we present a way of automatically devising collections of formally annotated videos by semantically parsing the natural language annotations in existing video captioning datasets. 


\subsection{Semantically Parsing Natural Language Annotations}

We used the FRED machine reading service [7] to parse natural language annotations into linked knowledge graphs. For each sentence, a call is made to the FRED service, and an RDF response is received. The requests made specify the flag wsd in order to indicate FRED to perform word sense disambiguation, and thus to provide alignments to entities in the Framester linked lexical knowledge base [6] whenever possible.

Figure 4 shows three frames extracted from a video in the MSVD dataset, together with the FRED output graph for its captioning sentence.

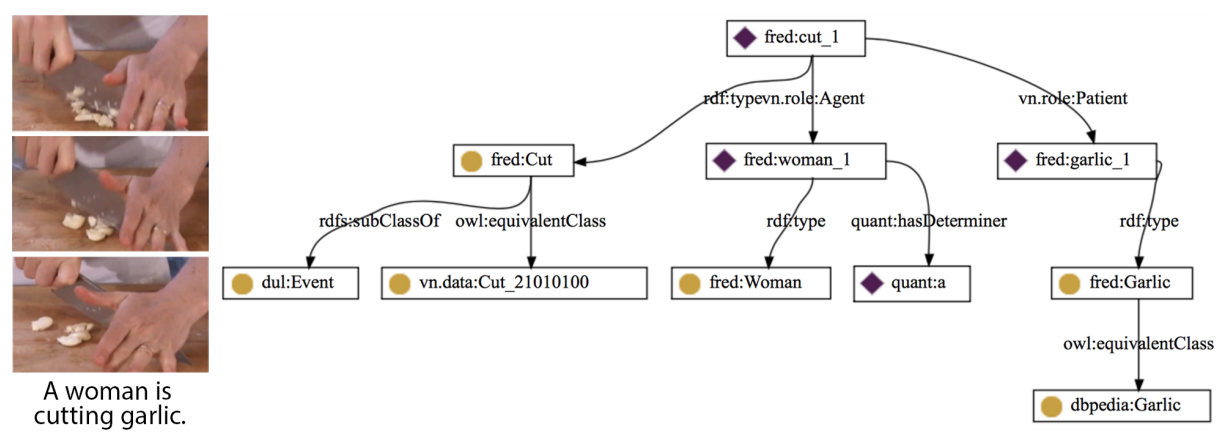

Fig. 4. FRED parsed video annotation example

\subsection{Post-Processing the Devised Formal Annotations}

Each knowledge graph output by FRED contains edges that define relationships between individuals (e.g., (fred:cut_1, vn.role:Agent, fred:woman_1)), type predicates (e.g., (fred:garlic_1, rdf:type, fred:Garlic)), and ontological rules (e.g., (fred:Garlic, owl:equivalentClass, dbpedia:Garlic)). Due to the way the graphs are generated by FRED, a certain amount of redundant information is contained in each of them (e.g., every graph that contains the individual fred:garlic_1 will also contain the type predicate (fred:garlic_1, rdf:type, fred:Garlic)).

In order to simplify the task of learning to map videos to such graphs, we post-process the formal annotations output by FRED. This involves isolating the type predicates and the ontological rules from each RDF annotation, in order to extract an underlying linked ontology for the entire dataset. The extracted facts can be inferred at a later stage by reasoning in the resulting underlying ontology.

Merging Identical Entities and Isolating the Ontological Rules. The main source of redundant background information in the generated graphs is represented by the ontological rules inserted by FRED in order to align the entities identified 
to entries in Framester. Equivalent concepts linked by the owl:equivalentClass predicate, as well as equivalent individuals linked by the owl:sameAs relation are merged together. The other ontological rules (e.g., rdf:subClassOf rules) are isolated in an external ontology. Figure 5 describes the result of performing this processing step on the annotation graph given in Figure 4. Notice that at this point, every edge in the graph has at least one individual at one of its ends.

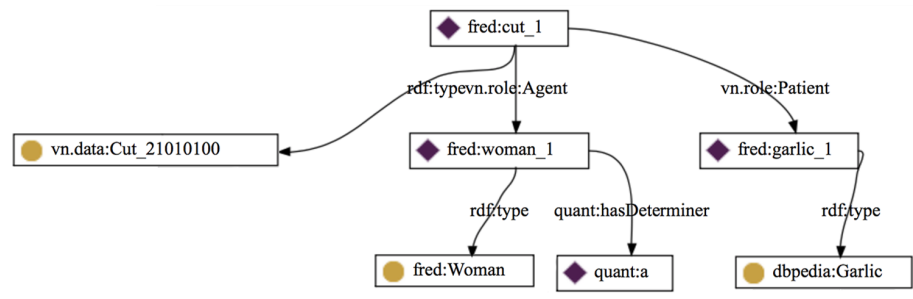

Fig. 5. Example result of isolating the ontological rules

Isolating the Type Predicates. The type specifications for each individual are part of the background ontological knowledge applicable to the entire dataset, and should thus be extracted to reduce the complexity of the annotation graphs. The result of isolating the type predicates on the example graph is given in Figure 6 .

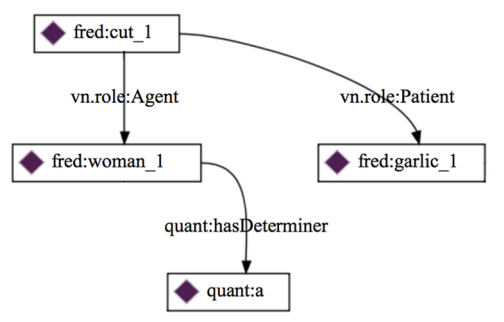

Fig. 6. Example result of isolating the type predicates

Dealing with Type Conflicts. Due to the particular way in which FRED performs Skolemization on the first-order predicate variables that it identifies along its parsing steps [7], it might be the case that the same individual is typed differently in two different formal annotations. An immediate example scenario is the one of using a homonym, such as "lie", to name an individual; the extracted individual fred:lie_1 could then be typed as a WordNet noun synset, or as an unrelated VerbNet class. Since the convention made earlier is that types are extracted as background knowledge (and are hence not to be handled by the video annotation 
models), there is a need to solve these conflicts, in order to avoid distorting the semantics of a video when later expanding a compressed annotation. This is achieved by extending the set of individuals in the ontology to distinguish between those with conflicting types.

\section{Formal Video Captioning Models}

In this section, we present three neural network models for learning structured video descriptions. The first one maps videos to sets of facts, while the second and the third one are based on adapting natural language annotation models.

\subsection{Mapping Videos to Sets of Facts}

There is a one-to-one correspondence between knowledge graphs and sets of facts, and hence, learning to formally annotate videos with knowledge graphs is equivalent to learning to map videos to such sets of facts, i.e., solving multilabel classification for videos, where the possible labels are the facts used in the training annotations.

The first formal annotation model explored by this work attempts to map sequences of frames to sets of facts by using a neural network architecture formed of a CNN-RNN temporal encoder, followed by a multi-label classifier network. Considering the high semantic dependency between the facts in an annotation that would need to be modelled, this solution involves training a single classifier instead of one for each label. The approach is motivated by the results presented in [10], which highlight the superiority of single classifiers when mapping documents to large sets of inter-dependent labels.

Figure 7 describes the design of the proposed neural network model. The layer sizes in the labelling part of the network are given for exemplification purposes and need to be tuned according to the number of facts in the training data, and to their frequency distribution.

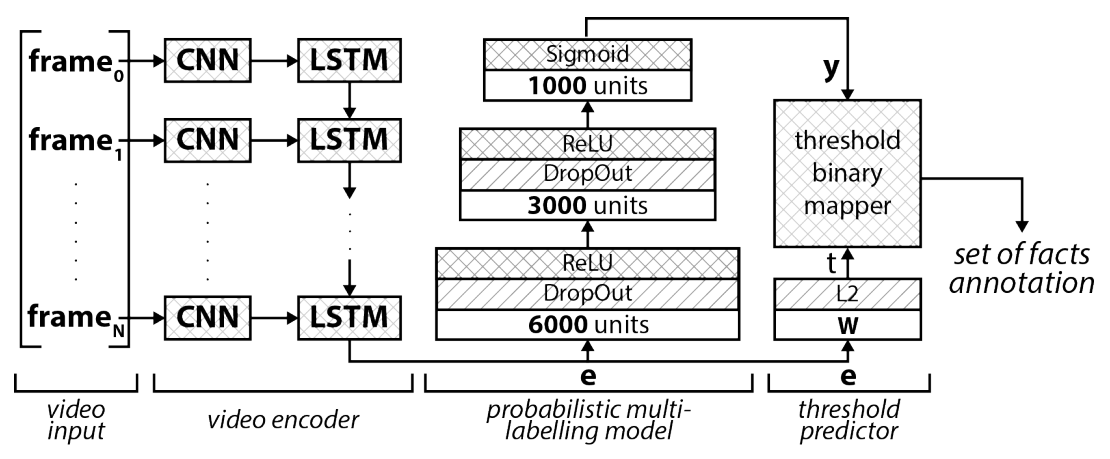

Fig. 7. Video to set-of-facts mapping model 
Video Temporal Encoder. For processing the frame level information, the model uses an encoder of the type depicted in Figure 2, taking the LSTM state at time $N$ as the video encoding (as suggested in [11]). Each frame is initially passed through a pretrained version of the VGG-19 image recognition model [15], and at each time step, the activations in the last hidden layer of the CNN are input by a 2-layer standard LSTM RNN using 1500 units.

Multi-Label Classification Network. For solving the multi-label classification problem of mapping the encoded video to a set of facts, the model employs a 2 -stage solution originally suggested in [10]. First, it learns a probabilistic model that outputs a distribution $\boldsymbol{y}_{i} \in[0,1]^{L}$ over the set of labelling facts (where $L$ is the number of distinct facts used to label training videos). This is achieved by employing a standard feed-forward neural network composed of two hidden layers using ReLU activations, and an output layer made of Sigmoid units. Dropout [16] is employed at the hidden layers for regularisation. Given $M$ training video encodings $\boldsymbol{e}_{i}$ and ground labellings $\overline{\boldsymbol{y}}_{i} \in\{0,1\}^{L}$, the model aims to minimize the cross-entropy loss

$$
c \equiv-\sum_{i=1}^{M}\left(\overline{\boldsymbol{y}}_{i} \log \boldsymbol{y}_{i}+\left(1-\overline{\boldsymbol{y}}_{i}\right) \log \left(1-\boldsymbol{y}_{i}\right)\right) .
$$

Transforming the obtained probability distribution to a binary vector encoding of a set is done via learning a threshold $t_{i}$ for each video, sorting the set of labels by the output probabilities $\boldsymbol{y}_{i}$, and then mapping the values above $t$ to 1 , and the ones below to 0 . The thresholds are learned using an L2-regularised linear regression model $t_{i}=\boldsymbol{\theta}^{T} \boldsymbol{e}_{i}$ with loss

$$
l \equiv \frac{1}{2 M} \sum_{i=1}^{M}\left(\boldsymbol{\theta}^{T} \boldsymbol{e}_{i}-\bar{t}_{i}\right)^{2}+\frac{\lambda}{2}\|\boldsymbol{\theta}\|^{2} .
$$

The ground threshold values $\bar{t}_{i}$ are obtained by using the generated probability distributions $\boldsymbol{y}_{i}$ for the training data, and for each data-point $i$, searching for the value $\bar{t}_{i}$ that maximises the $\mathrm{F}$ score of the generated set when mapping the distribution to a binary vector.

The probabilistic labelling model is trained end-to-end, together with the encoding RNN. The threshold predictor is learned in isolation in a second phase, using the already trained video encoder and probabilistic multi-labeller.

\subsection{Adapting a Natural Language Annotation Model}

As indicated in Section 2.2, there has been significant work done towards solving the problem of video captioning. Naturally, captioning models could be adapted to map videos to sequences of arbitrary entities, and doing so would mean benefiting from the important progresses made towards modelling and incorporating memory and attention into them. 
Inspired by [20], which proposes a solution for performing multi-label image classification using RNNs in order to model the semantic dependence between labels, we attempt to encode the annotating knowledge graphs as sequences and then train standard video captioning models over the obtained encodings. The next two models proposed use the same neural network architecture as the one introduced for natural language video captioning in [5] and briefly discussed in Section 2.2, up to a set of fine-tuned hyper-parameters. While this approach may seem counter-intuitive at first sight, since sequences are intrinsically ordered, while graphs are not, we explore a few variants for mapping knowledge graphs to ordered representations.

Knowledge Graphs as Sequences of Facts. The first encoding explored follows from the one based on binary vectors for representing subsets of facts used in the multi-label classifier model. The set of $L$ facts mentioned in the training annotations is turned into a vocabulary, and the graphs are then represented as ordered sequences of elements in this vocabulary.

The two orderings between facts that we experiment with are the lexicographic one, and the one of the frequency of a fact inside the corpus of training formal annotations. Comparing the two orderings, there is a very negligible performance between them (this matches the experimental results reported in [20] for multi-label image classification). However, both orderings significantly outperform randomly shuffling the sequences; in the latter case, the captioning model is virtually not trainable.

Knowledge Graphs as Sequences of Entities. Using sequences of facts as encodings has the disadvantage of a very large vocabulary to cover the large set of facts in the training corpus. At the cost of modelling longer sequences, but benefiting from a compressed vocabulary, the second proposal for encoding knowledge graphs is as sequences of separated, explicitly represented triples of entities. An example encoding for the set of facts

$$
\{(\text { man }, \text { sitsOn }, \text { bench }),(\text { woman }, \text { sitsOn, bench })\}
$$

would be "man sitsOn bench | woman sitsOn bench". In this variant, a vocabulary is extracted from the set of entities mentioned in the training annotations.

Similarly to the previous encoding, there is a need for inserting order into the sequences. For this encoding, we have chosen to format each triple of entities using the template "subject predicate object", and then lexicographically sort the triples of entities in the sequence.

\section{Experimental Evaluation}

In this section, we experimentally evaluate the three devised neural architectures for learning structured video descriptions. We first describe the evaluation methodology and then provide quantitative and qualitative evaluations. We highlight the potential of the devised models, indicating that they manage to capture 
the semantic content in a video scene with an accuracy that is similar to the one of a state-of-the-art natural language captioning model.

\subsection{Evaluation Methodology}

The three formal video annotation models suggested in Section 4 are evaluated by employing the same precision, recall, and F-score equations as the ones used by SPICE to measure the similarity between a predicted annotation graph and a corpus of reference ones. Since the graphs in the dataset of formally annotated videos (referred to as $M S V D-f$ below) have been post-processed, the semantically equivalent entities have already been merged; thus, when measuring the semantic overlap, the entities in the graphs are aligned using exact matching.

By using the same scoring strategy as SPICE (up to the parsing mechanisms employed), the semantic capturing performance of the models proposed can be comparatively evaluated against the natural language captioning baselines. This is essential, considering the absence in the literature of other structured annotation algorithms and datasets to compare the recorded results against.

The results presented below have been computed by analyzing the predicted annotation graphs directly, before expanding them using the extracted background knowledge, to focus the analysis on the models' performance. The recorded scores have all been scaled to the 0-100 domain in order to allow for cross-comparisons. All the models analyzed below have been trained for 24 hours using a single Tesla P100 16 GB GPU on the JADE Tier-2 high-performance computing facility. In order to obtain test scores that can be compared to the other approaches in the literature on video captioning, we have followed the same training/validation/test split as the one used in [5] for both MSVD and MSVD-f.

\subsection{Benchmarking Results}

Natural Language Baselines. Before evaluating the formal annotation models, a baseline is set by training and evaluating the video captioning model proposed and open-sourced in [5] on MSVD. The test scores are listed in Table 1 and have been checked to be in line with the ones reported in [5].

It is noticeable that the SPICE score is an order of magnitude lower than the other reported metrics. This is due to the reference sets of captions being semantically diverse, which, in combination with the design choice made for SPICE to evaluate the recall by considering the union of all reference scene graphs $(G(S))$, leads to lower scores. To better contextualise the results, the average SPICE score for picking one of the reference annotations as a prediction on the test fold of MSVD is 12.63.

As expected, the evaluation results of the structured video annotation models on MSVD-f show a comparably low recall as well (and hence, a low F-score), as described below. 
Table 1. Natural language captioning baseline performance

\begin{tabular}{lllll}
\hline BLEU-1 & BLEU-4 & METEOR & CIDEr & SPICE \\
\hline 75.43 & 43.03 & 30.21 & 59.85 & 5.03
\end{tabular}

Multi-Label Classifier (MLC). The first formal annotation model evaluated is the multi-label video classifier introduced in Section 4.1. The model has been trained after choosing the hyper-parameter $L$ and restricting the training labelling sets to subsets of the most frequent $L$ facts. Iteratively increasing $L$ gave the best validation results for $L=1000$ in the case of both datasets. Table 2 lists the test scores recorded for this model.

It is noticeable that the precision score is an order of magnitude higher than the recall score. This is expected considering the semantic diversity of the natural language annotations in MSVD, as discussed before.

Table 2. Multi-label classifier F-score

\begin{tabular}{lll}
\hline Precision & Recall & F-score \\
\hline 25.6 & 1.96 & 3.68
\end{tabular}

Video to Sequence Models (Vid2Facts and Vid2Ents). Moving on to the video to sequence models introduced in Section 4.2, Table 3 presents the test scores for the models trained on annotation graphs encoded as sequences of facts (Vid2Facts), and on the ones encoded as sequences of entities (Vid2Ents).

For Vid2Facts, although BLEU-1 records a $23 \%$ facts overlap, and the average precision recorded is $34.95 \%$ (the precision formula used awards partial credit for facts that correctly identify the objects in a scene, even when they are wrongly related), the SPICE score is brought down by a low average recall of only $3.10 \%$.

For Vid2Ents, the high n-gram overlap scores, together with a relatively high average precision of $41.41 \%$ indicate that the model is able to capture a significant part of the combined semantics of the reference annotation graphs. Again, the F-score is brought down by a low corpus-level average recall of $3.36 \%$.

Table 3. Video to sequence-of-facts test performance

\begin{tabular}{llllllll}
\hline Model & BLEU-1 & BLEU-4 & METEOR & CIDEr & Pre. & Rec. & F-score \\
\hline Vid2Facts & 23.00 & 12.10 & 9.40 & 20.80 & 34.98 & 3.10 & 5.58 \\
Vid2Ents & 65.60 & 35.40 & 29.50 & 80.80 & 41.41 & 3.36 & 6.11
\end{tabular}

Summary of the Recorded Results. The results presented above indicate that the formal annotation models proposed manage to partially capture video semantic 
content in a structured manner. It has also been shown that their performance, measured by the proposed F-score, is in line with the SPICE performance of a state-of-the-art natural language baseline model.

Comparing the three proposed methods, it is apparent that the adapted natural language models (Vid2Facts and Vid2Ents) outperform MLC on both datasets. Table 4 gives one insight into why this might be the case, showing that the MLC model outputs smaller graphs, further affecting its recall scores. This suggests that the threshold predictor used for mapping probability distributions to sets could be a potential bottleneck, and future attempts to improve performance could focus on better modelling it.

Table 4. Average annotation graph sizes

\begin{tabular}{llll}
\hline Ground & MLC & Vid2Ents & Vid2Facts \\
\hline 3.61 & 2.60 & 3.27 & 3.68
\end{tabular}

\subsection{Qualitative Analysis}

To complete the evaluation of the models proposed by this report, Figure 8 presents four test scenarios extracted from the MSVD dataset, each containing a selection of three reference natural language annotations, together with the predictions made by the baseline natural language model, and by the three formal annotation models discussed (in the form of knowledge graphs); each prediction has attached its SPICE/F score.

The given examples reveal the discussed semantic diversity of the reference annotations in MSVD, with the recorded F scores being biased towards the larger annotations that overlap more with the union of the reference graphs (e.g., the annotation that describes both a man cooking, and the fact that chicken is being cooked, receives a higher score than the one that describes the more specific action of a man putting seasoning in a plastic container).

The last example highlights the impact on performance of the machine reader used to semantically parse datasets: the term rodent is consistently incorrectly parsed by FRED as a "VeryShortRodeoRide animal" in the training dataset.

Finally, the selection of examples in Figure 8 highlights the similar ability of the four annotation models to capture the semantic content of the reference corpora. Both the natural language and the formal annotation models usually output well-structured captions that are semantically close to the references.

\section{Summary and Outlook}

This paper was focused on developing a solution for automated video knowledge extraction in the form of linked graphs. In the absence of existing public formal video annotation datasets at the time of writing, we first introduced 


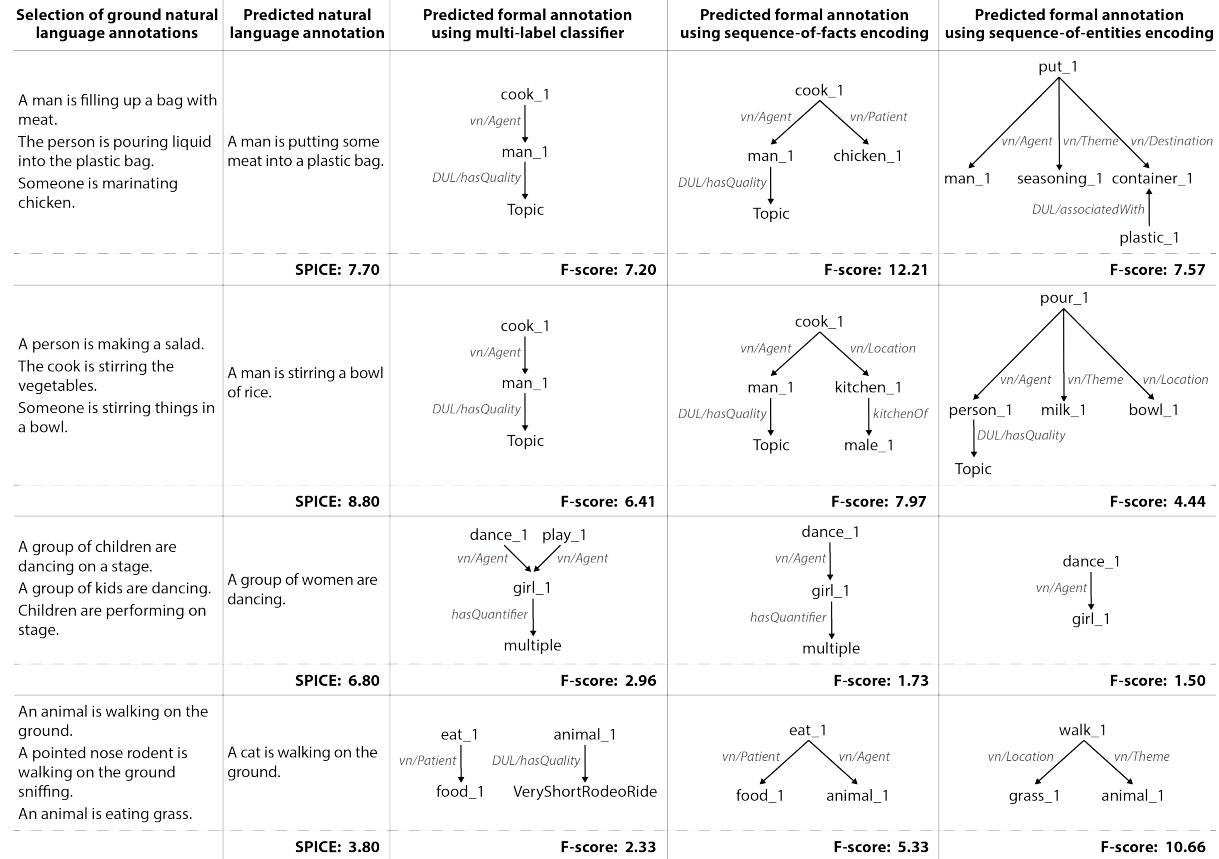

Fig. 8. Qualitative analysis

MSVD-f, a formal video annotation dataset obtained by semantically parsing and post-processing a natural language video captioning corpus. The main presented contribution was then designing three formal video captioning models and evaluating them on the devised dataset. The best performing two of them were shown to be able to describe the semantic content of the test videos with a similar degree of accuracy to the one of state-of-the-art natural language captioning models. To our knowledge, no formal video captioning solution had been publicly presented and evaluated at the time of writing. Some interesting directions for future research are summarized below.

The semantic parsing mechanism used to devise MSVD-f has been chosen to provide alignments to linked background knowledge bases, to enable reasoning on top of the predicted annotation graphs, once these are generated. However, the models proposed by this report do not input these alignments. A promising direction towards improving the video knowledge extraction performance would be to leverage the background information in the annotation prediction phase. Reasoning over partially generated graphs or over a set of candidate graphs, while employing commonsense knowledge may increase the annotation performance by iteratively expanding the set of observed facts at each prediction step, and by early detecting and isolating potential semantic anomalies.

The performance metrics for the proposed models (see Section 5.2) are interpreted assuming that the reference formal annotations in the two automatically 
devised datasets are correct. While making such an assumption is reasonable when comparing the designed models against each other, assessing their realworld performance requires that the accuracy of the reference annotations used for training is factored in. Future work on video knowledge extraction could consider exploring more accurate semantic parsers, and ultimately employing human judgement, in order to improve the evaluation accuracy and the relevance of the obtained models.

The devised dataset is composed of small, summarizing annotation graphs (4.3 triples on average) that fail to capture the complete semantics of most video scenes. A proof in this sense is the high semantic diversity of the reference annotation corpora in MSVD; as reference annotations, they are all valid, but describe disjoint semantics by focusing on different aspects in a video scene. One would expect video knowledge extraction models to generate dense structured descriptions that fully capture the semantics of video scenes, similarly to how the dense structured annotations in the Visual Genome dataset [9] capture image/frame semantics. An immediate step towards training such models would be to automatically generate datasets of dense formal video annotations by joining the reference captions of each video into single annotation graphs.

Acknowledgements. This work was supported by The Alan Turing Institute under the UK EPSRC grant EP/N510129/1, and by the EPSRC grants $\mathrm{EP} / \mathrm{R} 013667 / 1, \mathrm{EP} / \mathrm{L} 012138 / 1$, and EP/M025268/1.

We are grateful to the authors of [5] for open-sourcing and documenting a very powerful video captioning model. We thank the STLAB team for making the FRED machine reader [7] publicly available, and acknowledge the use of the Microsoft Video Description (MSVD) dataset [3]. Finally, we thank Oana TifreaMarciuska, Patrick Hohenecker, and Ruomei Yan for insightful discussions on semantic parsing, encoding knowledge graphs, and deep neural video captioning.

All the computation required for carrying out this work has been performed using the JADE Tier-2 high-performance computing facility.

\section{References}

1. Anderson, P., Fernando, B., Johnson, M., Gould, S.: SPICE: Semantic propositional image caption evaluation. In: Proc. of ECCV. pp. 382-398 (2016)

2. Auer, S., Bizer, C., Kobilarov, G., Lehmann, J., Cyganiak, R., Ives, Z.: DBpedia: A nucleus for a Web of Open Data. In: The Semantic Web, pp. 722-735 (2007)

3. Chen, D.L., Dolan, W.B.: Collecting highly parallel data for paraphrase evaluation. In: Proc. of ACL. Volume 1. pp. 190-200 (2011)

4. Denkowski, M., Lavie, A.: Meteor Universal: Language specific translation evaluation for any target language. In: Proc. of WMT. pp. 376-380 (2014)

5. Fakoor, R., Mohamed, A.R., Mitchell, M., Kang, S.B., Kohli, P.: Memoryaugmented attention modelling for videos. arXiv:1611.02261 (2016)

6. Gangemi, A., Alam, M., Asprino, L., Presutti, V., Recupero, D.R.: Framester: A wide coverage linguistic linked data hub. In: Proc. of EKAW. pp. 239-254 (2016) 
7. Gangemi, A., Presutti, V., Reforgiato Recupero, D., Nuzzolese, A.G., Draicchio, F., Mongiovì, M.: Semantic Web machine reading with FRED. Semantic Web 8(6), 873-893 (2017)

8. Johnson, J., Hariharan, B., van der Maaten, L., Fei-Fei, L., Zitnick, C.L., Girshick, R.: CLEVR: A diagnostic dataset for compositional language and elementary visual reasoning. In: Proc. of IEEE CVPR. pp. 1988-1997 (2017)

9. Krishna, R., Zhu, Y., Groth, O., Johnson, J., Hata, K., Kravitz, J., Chen, S., Kalantidis, Y., Li, L.J., Shamma, D.A., Bernstein, M., Fei-Fei, L.: Visual Genome: Connecting language and vision using crowdsourced dense image annotations. arXiv:1602.07332 (2016)

10. Nam, J., Kim, J., Mencía, E.L., Gurevych, I., Fürnkranz, J.: Large-scale multilabel text classification-Revisiting neural networks. In: Proc. of ECML PKDD. pp. 437-452 (2014)

11. Ng, J.Y.H., Hausknecht, M., Vijayanarasimhan, S., Vinyals, O., Monga, R., Toderici, G.: Beyond short snippets: Deep networks for video classification. In: Proc. of IEEE CVPR. pp. 4694-4702 (2015)

12. Papineni, K., Roukos, S., Ward, T., Zhu, W.J.: BLEU: A method for automatic evaluation of machine translation. In: Proc. of ACL. pp. 311-318 (2002)

13. Schuster, S., Krishna, R., Chang, A., Fei-Fei, L., Manning, C.D.: Generating semantically precise scene graphs from textual descriptions for improved image retrieval. In: Proc. of VL. pp. 70-80 (2015)

14. Sharma, S., Kiros, R., Salakhutdinov, R.: Action recognition using visual attention. arXiv:1511.04119 (2015)

15. Simonyan, K., Zisserman, A.: Very deep convolutional networks for large-scale image recognition. arXiv:1409.1556 (2014)

16. Srivastava, N., Hinton, G., Krizhevsky, A., Sutskever, I., Salakhutdinov, R.: Dropout: A simple way to prevent neural networks from overfitting. JMLR 15(1), 1929-1958 (2014)

17. Vedantam, R., Lawrence Zitnick, C., Parikh, D.: CIDEr: Consensus-based image description evaluation. In: Proc. of IEEE CVPR. pp. 4566-4575 (2015)

18. Vinyals, O., Toshev, A., Bengio, S., Erhan, D.: Show and Tell: A neural image caption generator. In: Proc. of IEE CVPR. pp. 3156-3164 (2015)

19. Wang, D., Song, D.: Video captioning with semantic information from the knowledge base. In: Proc. of IEEE ICBK. pp. 224-229 (2017)

20. Wang, J., Yang, Y., Mao, J., Huang, Z., Huang, C., Xu, W.: CNN-RNN: A unified framework for multi-label image classification. In: Proc. of IEEE CVPR. pp. 22852294 (2016)

21. Wu, Q., Shen, C., Liu, L., Dick, A., van den Hengel, A.: What value do explicit high level concepts have in vision to language problems? In: Proc. of IEEE CVPR. pp. 203-212 (2016)

22. Wu, Z., Yao, T., Fu, Y., Jiang, Y.G.: Deep learning for video classification and captioning. arXiv:1609.06782 (2016) 